Article

\title{
Process Modelling of Geothermal Drilling System Using Digital Twin for Real-Time Monitoring and Control
}

\author{
Nahashon O. Osinde ${ }^{1}$ () Jean B. Byiringiro ${ }^{1, *(\mathbb{D}}$, Michael M. Gichane ${ }^{1}$ (i) and Hasan Smajic $^{2}$ (i) \\ 1 Siemens Mechatronic Training Center, Dedan Kimathi University of Technology, 10100 Nyeri, Kenya \\ 2 Faculty of Vehicle Systems and Production, Institute of Production (IFP), Technology Arts Science TH Koln, \\ 50678 Köln, Germany \\ * Correspondence: jean.bosco@dkut.ac.ke; Tel.: +254-716-150-627
}

Received: 16 July 2019; Accepted: 15 August 2019; Published: 17 August 2019

\begin{abstract}
Currently, Kenya supplies its energy demand predominantly through hydroelectric power, which fluctuates due to poor and unpredictable rainfall in particular years. Geothermal energy is proposed as a clean and reliable energy source in meeting Kenya's increasing energy demand. During geothermal drilling operations, disruptions due to tool wear and breakages increases the cost of operation significantly. Some of these causes can be mitigated by real-time monitoring of the tool head during operations. This paper presents the design and implementation of a digital twin model of a drilling tool head, represented as a section of a mechatronic assembly system. The system was modelled in Siemens NX and programmed via the TIA portal using S7 1200 PLC. The digital model was programmed to exactly match the operations of the physical system using OPC (open platform communications) standards. These operations were verified through the motion study by simultaneous running of the assembly system and digital twin model. The study results substantiate that a digital twin model of a geothermal drilling operation can closely mimic the physical operation.
\end{abstract}

Keywords: geothermal drilling; digital twin; virtual model; open platform communication (OPC); real-time monitoring and control; Siemens NX

\section{Introduction}

The fourth industrial revolution also referred to as industrie 4.0 is the current trend in automation technology [1]. It encompasses digitalization, networking technologies, internet of things, cloud computing and cyber-physical systems. Digital twin is a key component in the realization of industrie 4.0. It involves the creation of a virtual representation of an existing physical system in cyberspace [2]. As discussed by Fei et al. [3], such a representation acts as an effective test-bed for implementing data fusion, machine learning and artificial intelligence to achieve real time control, monitoring and optimization. Digital twins are gaining popularity in research and industry as they can accurately represent the status, position or working conditions of their physical counterparts.

This study is geared at solving current geothermal drilling challenges through the implementation of a drilling process digital twin. The underground section of geothermal drilling involves a rotating tool head with water vents to allow the circulation of slurry used to clear debris from the drill tip. By attaching a torque sensor to the drill shaft and relaying such information to the digital twin, an accurate picture of the drill tip status can be obtained. Monitoring of drill torque fluctuations is an effective method of early fault detection and diagnosis. This will reduce the need to withdraw the drill tip from the borehole and subsequently save on cost and time losses due to drill halting. 
The initial step in the realization of a digital twin is the creation of a CAD model that is adequately programmed so as to closely synchronize with its physical counterpart. This study demonstrates the successful achievement of this concept.

\section{Literature Review}

\subsection{Energy and Economic Benefits of Pursuing Geothermal Drilling}

Energy is a key resource that is necessary for economic growth and development. As highlighted by the Government of Kenya energy development plan (2017-2037), Kenya has invested heavily in geothermal energy, which supplies close to $50 \%$ of Kenya's total power generation and the Kenyan government formed the Geothermal Development Company (GDC) to spearhead research and efforts in geothermal exploration. In order to achieve vision 2030, the energy demand in Kenya is projected to increase at a rate of $7 \%$ per annum. Currently, the total installed geothermal capacity in Kenya amounts to approximately $650 \mathrm{MW}$. According to geological surveys, Kenya's geothermal resource potential is close to 10,000 MW along its Rift Valley [4]. From these projections, geothermal energy is expected to be an essential resource in the future of the Kenyan power system. Technology and technical know-how to successfully exploit this resource is currently available in the country. However, high drilling cost, risks associated with drilling and long implementation periods have prevented fast growth in the sector.

\subsection{Geothermal Drilling Challenges}

Geothermal drilling has many similarities to oil and gas drilling, but there are many other different variables that make it more challenging. According to studies by Capuano et al. [5] , it has been evaluated that the cost of geothermal well development ranges from 4.5 to 5.5 million dollars per installed megawatt. Half the cost in well development is directed towards the drilling process. Some of the key contributors to the cost include: bit and stabilization cost, directional drilling cost, tools and on-site supervision, etc. [6].

Disruptions during the drilling process also greatly contribute to increased cost of well development. These usually occur when incidences such as bit breakage and drain pipe clogging result in the drilling tool being extracted for inspection and repair. These kinds of delays increase the cost implication since drilling cost is proportional to the total time spent in well development. The impact of drilling disruptions on cost is further supported by ARC Advisory Group estimations that project unplanned down-time leads to losses amounting to 1 trillion dollars a year [3]. During geothermal drilling, a typical drill pipe injection profile is as shown in Figure 1.

The profile consists of a drill pipe, surface and intermediate casings and terminates in a production hole where the geothermal energy is tapped [7]. Since the drilling operation is done underground, direct monitoring of the down-hole motor and the tool head is a challenge for the above ground drill team. When disruptions such as bit breakage, motor malfunction or pipe blockage occur, it necessitates the extraction of the drilling tool for inspection and repair. 


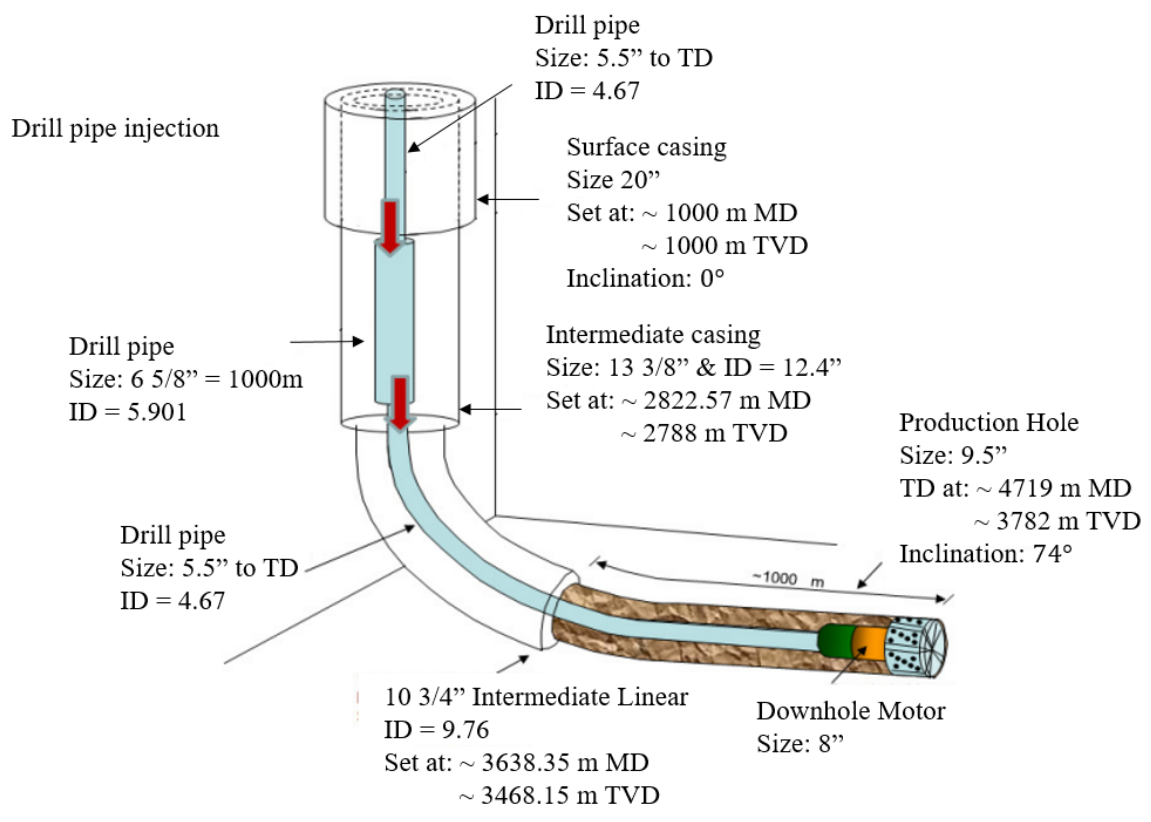

Figure 1. Drill pipe injection profile (courtesy of [8]).

\subsection{Current and Potential Solutions for Geothermal Cost Reduction}

As mentioned, drill bit replacement and repair contribute to the overall cost of geothermal drilling. Efforts in making geothermal drilling more economical have been researched, with scientists focusing on material selection and bit design in making stronger and more wear resistant tool heads and better methods of project planning and management. According to research by Miyazaki et al. [9], materials such as diamond bits and polycrystalline diamond (PDC) have been widely adopted in oil and gas drilling and research is ongoing on their suitability for geothermal application with promising preliminary research results. Drill bit designs also vary and include the conventional tri-cone roller and other innovations such as impregnated and dual-diameter bits as shown in Figure 2. These new bit designs last longer and reduce drilling, thus lowering the overall cost of well installation [4].

(a)

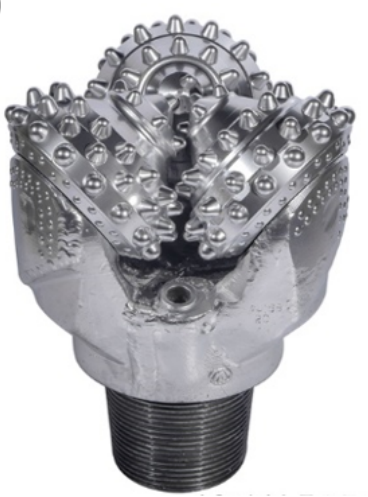

(b)

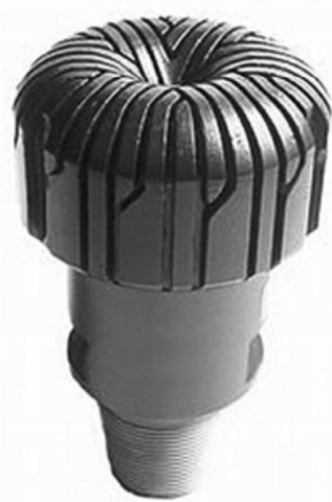

(c)

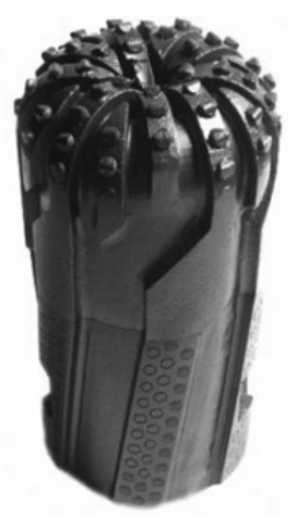

Figure 2. (a) tricone roller drilling bit; (b) impregnated drilling bit; (c) dual diameter bit [4]. 
Real-time monitoring and data acquisition through the use of embedded sensors can aid in establishing the status of the down-hole motor and drill tip. This information in digital form can enable experienced engineers to mitigate drilling disruptions and tool extraction for inspection or repair $[9,10]$. However, the concept of digital twin facilitates an inexperienced personnel to real-time monitor and switch control the drilling process by virtually looking at the dynamic behaviour of the drilling bit such as speed, feed, irregularities, etc. and decide to take measures which may lead to faster maintenance at reduced cost.

As highlighted, a digital twin is a virtual duplicate of a physical system but, with regards to well drilling, the use of a digital twin in operations has not been widely adopted [11]. This research aims to simulate a geothermal drilling operation where the virtual model is linked to its physical counterpart and real-time data obtained for drilling optimization. Access to actual geothermal drilling equipment suitable for experimentation was found to be difficult and hence the torquing station of a mechatronic assembly system was used as a representation of a typical drilling process.

\section{Design of the Digital Twin for the Torquing Station in Mechatronic Assembly System}

Generally, the implementation of a digital twin for a physical system involves the following components: Virtual Model, Communication Platform, PLC Programming Software and the Physical system as shown in Figure 3.

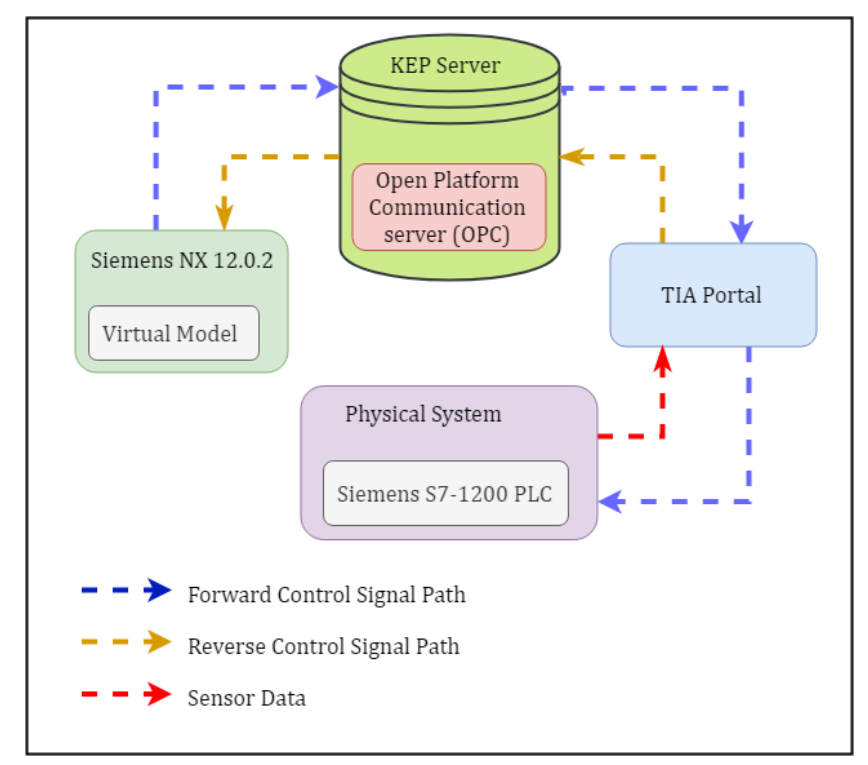

Figure 3. Block diagram of the digital twin implementation.

\subsection{Virtual Model}

A virtual model refers to a digital representation of a physical system. The digital twin should mimic its physical counter part in geometry and functionality. The design of the virtual model was done in Siemens NX 12.0.2 (Siemens PLM Software, Plano, Texas, U.S.). Siemens NX is a powerful software and is integrated with an application known as the Mechatronic Concept Designer (MCD). The MCD helps in assigning physical properties and motions to the CAD models which are similar to those of the actual physical system. The first step in the CAD implementation was acquiring accurate dimensions of the system and creating CAD models of the station components. The entire system was divided into several sub-assemblies. These included: the work piece, slide mechanism, torquing motor and tool, clamp assembly and control panel. Each of these sub-assemblies was drawn in Siemens $\mathrm{NX}$ and later these sub-assemblies were put together to form the final assembly. To enable motion simulation, the physics, material and position control properties were configured into the CAD models and relevant joints assigned to connect the various components of the sub-assemblies. 


\subsection{Communication Platform}

To ensure flow of data and control signals between the physical system and the virtual model, a suitable means of communication is required. An interoperability standard for secure and reliable exchange of data in industrial automation is the Open Platform Communication (OPC). These standards are facilitated via KEP server which provides client server communication. Through the KEP server, information can be sent and stored from the digital twin to the physical system and vice versa.

\subsection{PLC Programming Software-TIA Portal}

TIA refers to Total Integrated Automation portal. It is a Siemens specific platform for programming PLC hardware and enabling online communication, real-time monitoring and integration with other features i.e., Cloud Computing, Artificial Intelligence control, etc. The functionality/various process steps of the physical system are defined and sequenced in code through the use of ladder diagrams. These are then downloaded to the PLC.

\subsection{Physical System-Mechatronic Torquing Station}

The torquing station is part of a valve assembly system where a torquing tool is used to tighten the spool on the work-piece and ensure it is held in place. This torquing action is a similar operation to the drilling of a geothermal well. The tooling that torques the valve screw represents the geothermal drill tip while the actual torquing operation represents the actual drilling process. Figure 4 shows a flowchart that highlights the step sequence of the torquing station. Once an assembled valve is placed on the torquing station, it is clamped and held in place by the pneumatic cylinder. After securing the work-piece, the spool screw is tightened by the tooling for a period of $3 \mathrm{~s}$. With the torquing complete, the valve is un-clamped and transported to the end of travel (EOT) of the station. Figure 5 shows the actual torquing station that was modelled.

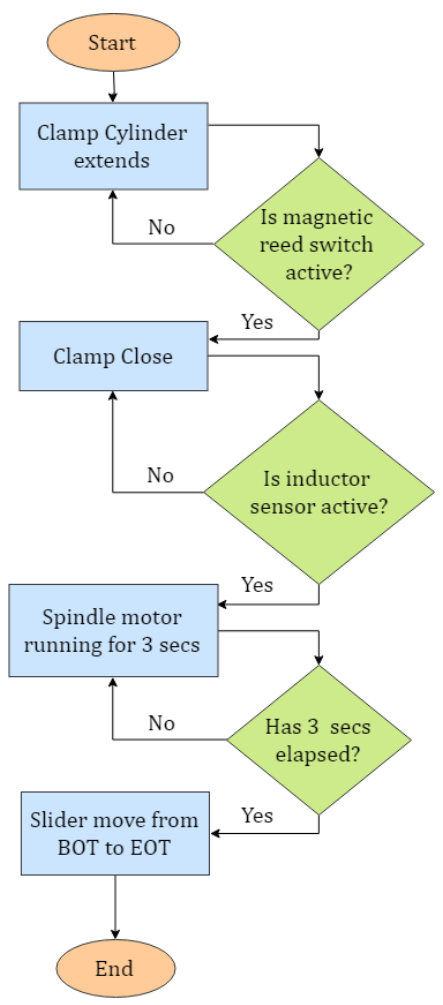

Figure 4. Flowchart illustrating the control sequence of the torquing station.

This torquing process can be run under three distinct operation modes: 
1. Automatic Mode: This is the normal autonomous system operation mode, where the machine runs without interference by the user.

2. Manual Mode: In the event, calibration, repair or debugging is required, the process sequence steps are controlled directly by the user who manually drives the process from one step to the next.

3. Reset Mode: During Automatic or Manual mode operation, the reset button can be pressed. This returns the system to its initial position.

As a safety feature of the system, an emergency stop button is incorporated. When pressed, all system operations cease until the system is reset.

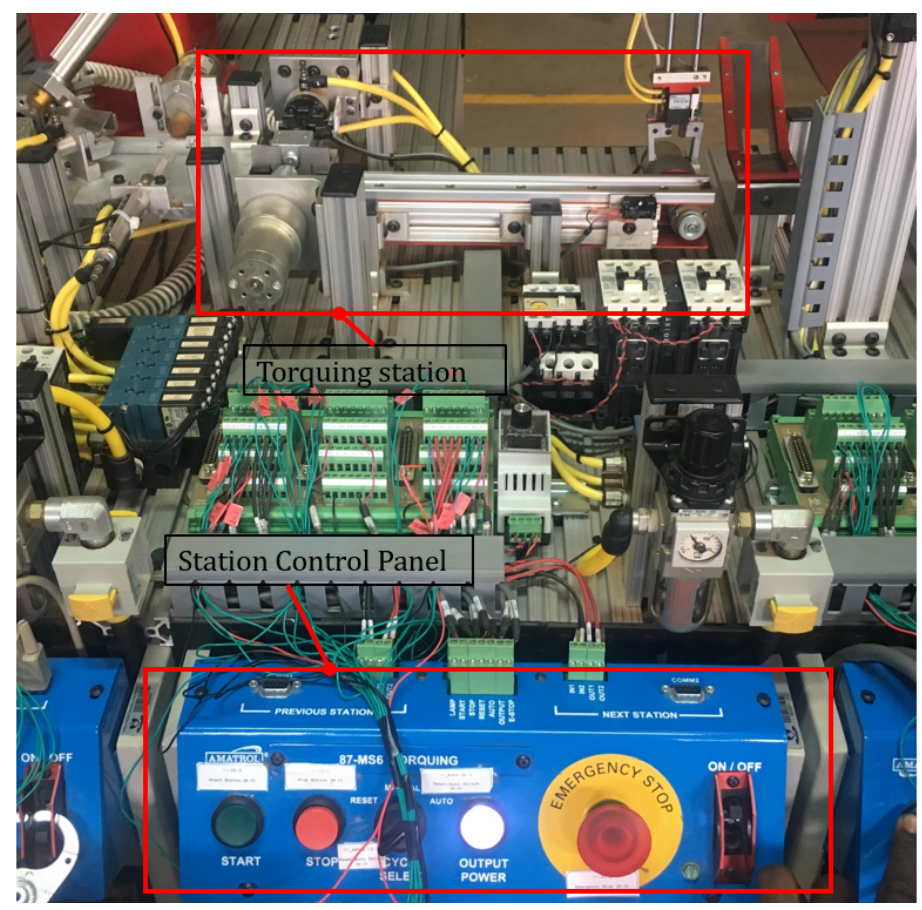

Figure 5. The physical set-up of the torquing station.

\section{Implementation of the Digital Twin of a Mechatronic Assembly System}

\subsection{Virtual Model—CAD Design}

Creating the virtual model involved designing the various sub systems and assembling them so that they replicate the actual physical system. This was done in Siemens NX where the various sub-systems-work-piece, slide mechanism,torquing motor, clamp assembly and panel-were implemented as shown in Figures 6 and 7. All of these sub assemblies were then combined into a final assembly as shown in Figure 8. After adequate configuration, each sub-assembly was tested to verify that it operated as intended. 


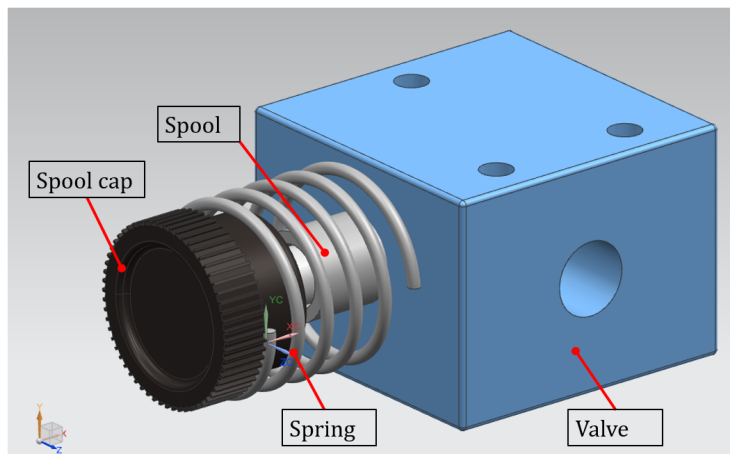

(a)

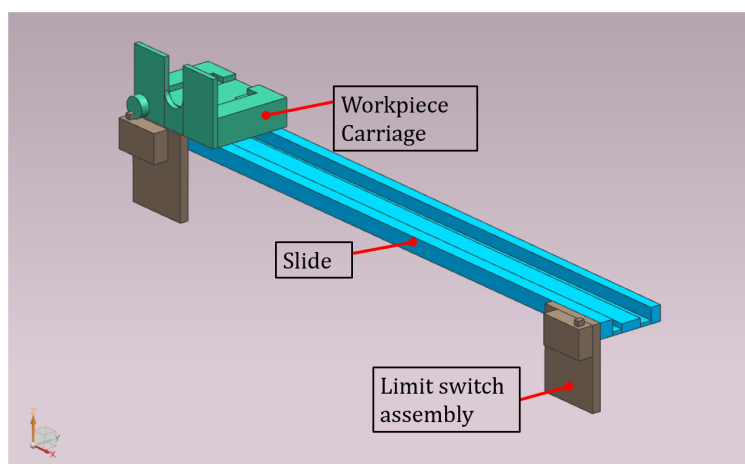

(b)

Figure 6. (a) work piece-valve assembly; (b) slide mechanism.

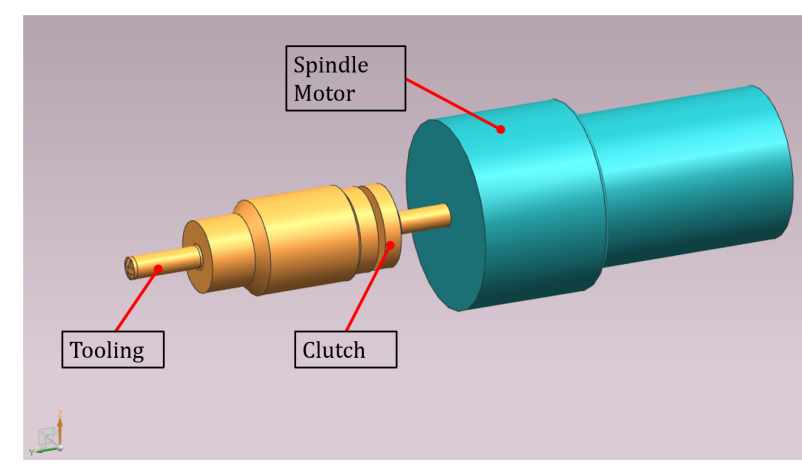

(a)

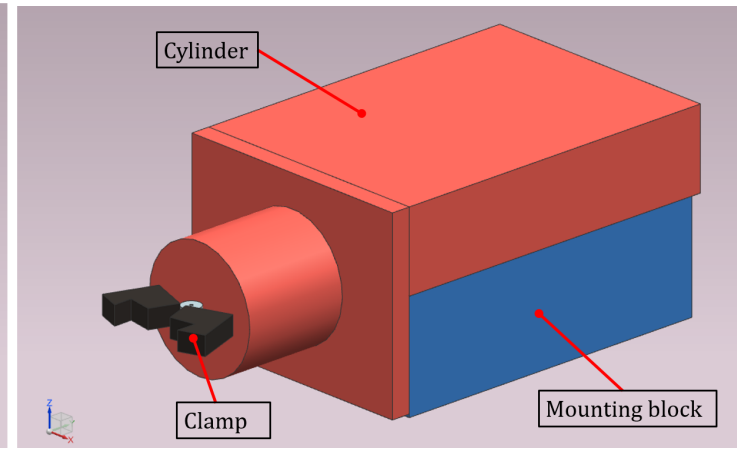

(b)

Figure 7. (a) torquing motor and tool; (b) clamp assembly.

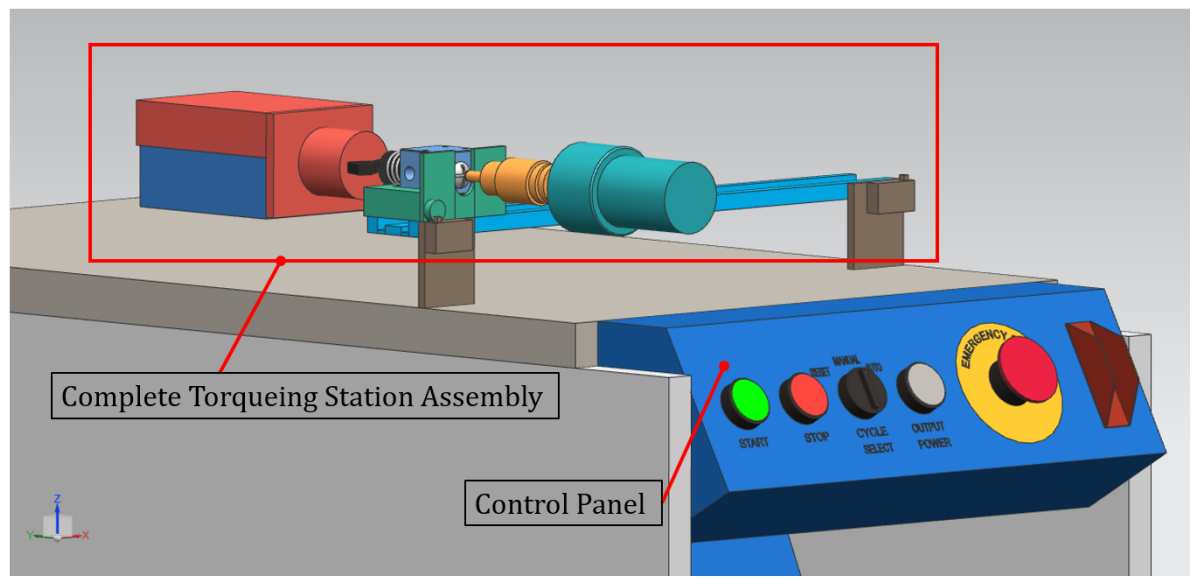

Figure 8. Final assembly of the torquing station.

\subsection{Communication}

Relaying of control signal and data between the digital twin and the physical system was achieved through the use of OPC standards via KEP server. Firstly, Siemens NX was configured to communicate with KEP-server via the External Signal Configuration (ESC) feature. The ESC feature facilitated the merger between control signals from the digital twin to an external source, which, in our case, was the TIA portal. The TIA portal connected to the KEP-server uses link tags that specify sensor and actuator control signals. These tags were then configured in the ESC feature, by assigning them similar names and addresses. This step completed the communication linkage between the digital twin and 
physical counterpart. The communication was then tested and the status of the links were deemed to be adequate as shown in Figure 9.

\begin{tabular}{|c|c|c|c|c|c|}
\hline \multicolumn{6}{|l|}{ Mapped Signals } \\
\hline Connection Name & MCD Signal Name & Direction & External Signal Name & Owner Component & है? \\
\hline$\checkmark$ SignalAdapter(1)_started_STARTED & started & $\leftarrow$ & Channel1.Device1.STARTED & $\wedge$ & \\
\hline$\checkmark$ SignalAdapter(1)_extended_EXTE... & extended & $\rightarrow$ & Channel1.Device1.EXTENDED & & \\
\hline$\checkmark$ SignalAdapter(1)_retracted_RETRA... & retracted & $\rightarrow$ & Channel1.Device1.RETRACTED & & \\
\hline$\checkmark$ SignalAdapter(1)_clamped_CLAM... & clamped & $\rightarrow$ & Channel1.Device1.CLAMPED & & \\
\hline$\checkmark$ SignalAdapter(1)_start_START & start & $\rightarrow$ & Channel1.Device1.START & & \\
\hline$\checkmark$ SignalAdapter(1)_stop_STOP & stop & $\rightarrow$ & Channel1.Device1.STOP & & \\
\hline$\checkmark$ SiqnalAdapter(1)_power_POWER & power & $\rightarrow$ & Channel1.Device1.POWER & $\checkmark$ & \\
\hline$<$ & & & & $>$ & \\
\hline
\end{tabular}

Figure 9. Mapping interface for the MCD and KEP-server signals.

\subsection{Control Sequence}

The process control sequence of the torquing station starts from extending the clamp cylinder while checking if the magnetic reed switch is active. If the clamp cylinder reaches its limit, the reed switch is activated and the clamp is then closed. Once completely closed, the inductive sensor becomes active and the torquing motor is run for $3 \mathrm{~s}$. After $3 \mathrm{~s}$, the torquing motor stops and the clamp releases and retracts. The work-piece is then transported from the beginning to the end of travel via the slider mechanism. Figures 10 and 11 show the integration of the cyber and physical station with switching control through TIA and NX programs and Figures 12-15 illustrate both the physical system and the digital twin at each step of the operation sequence.

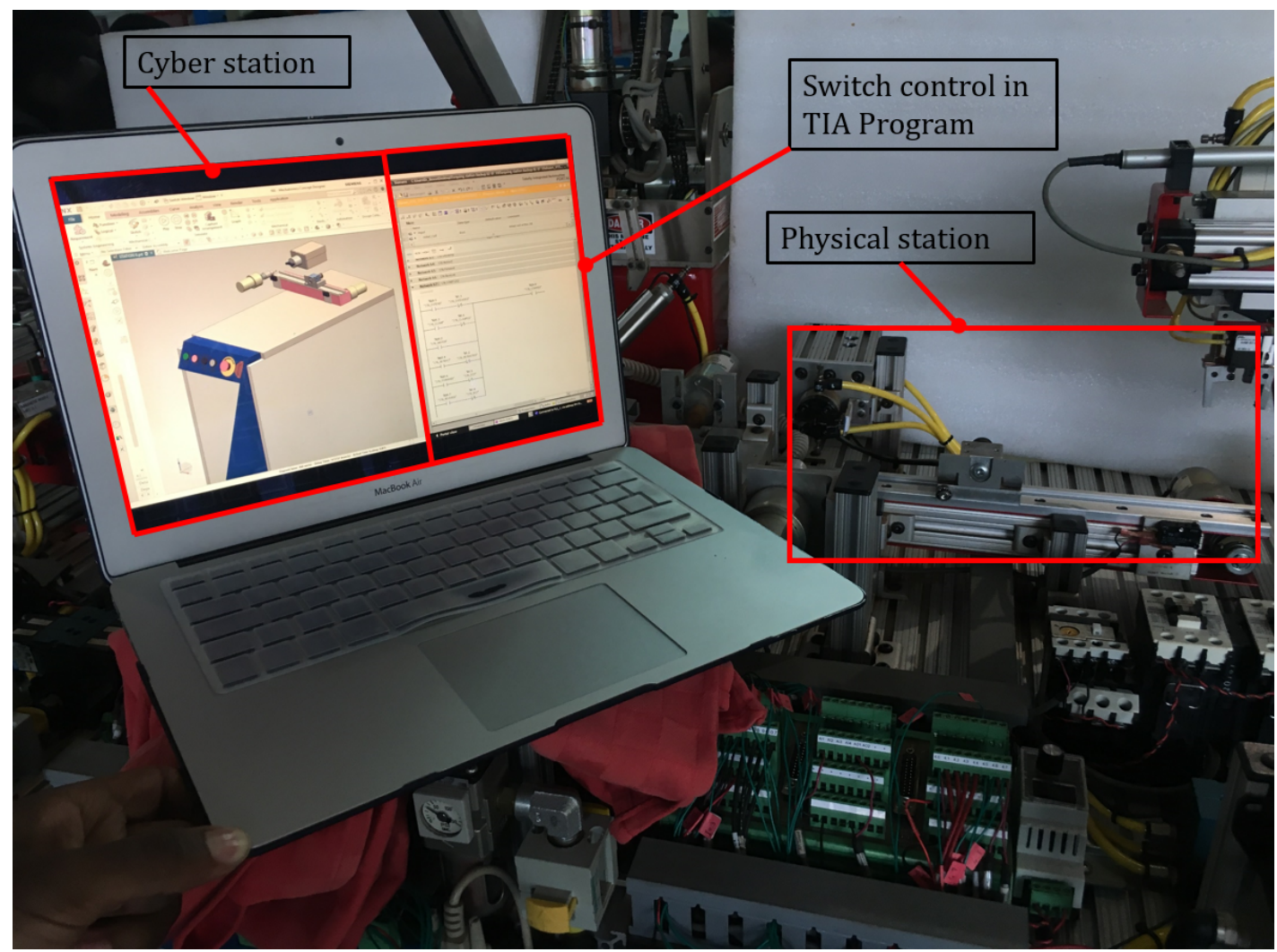

Figure 10. Integration of the cyber and physical station. 


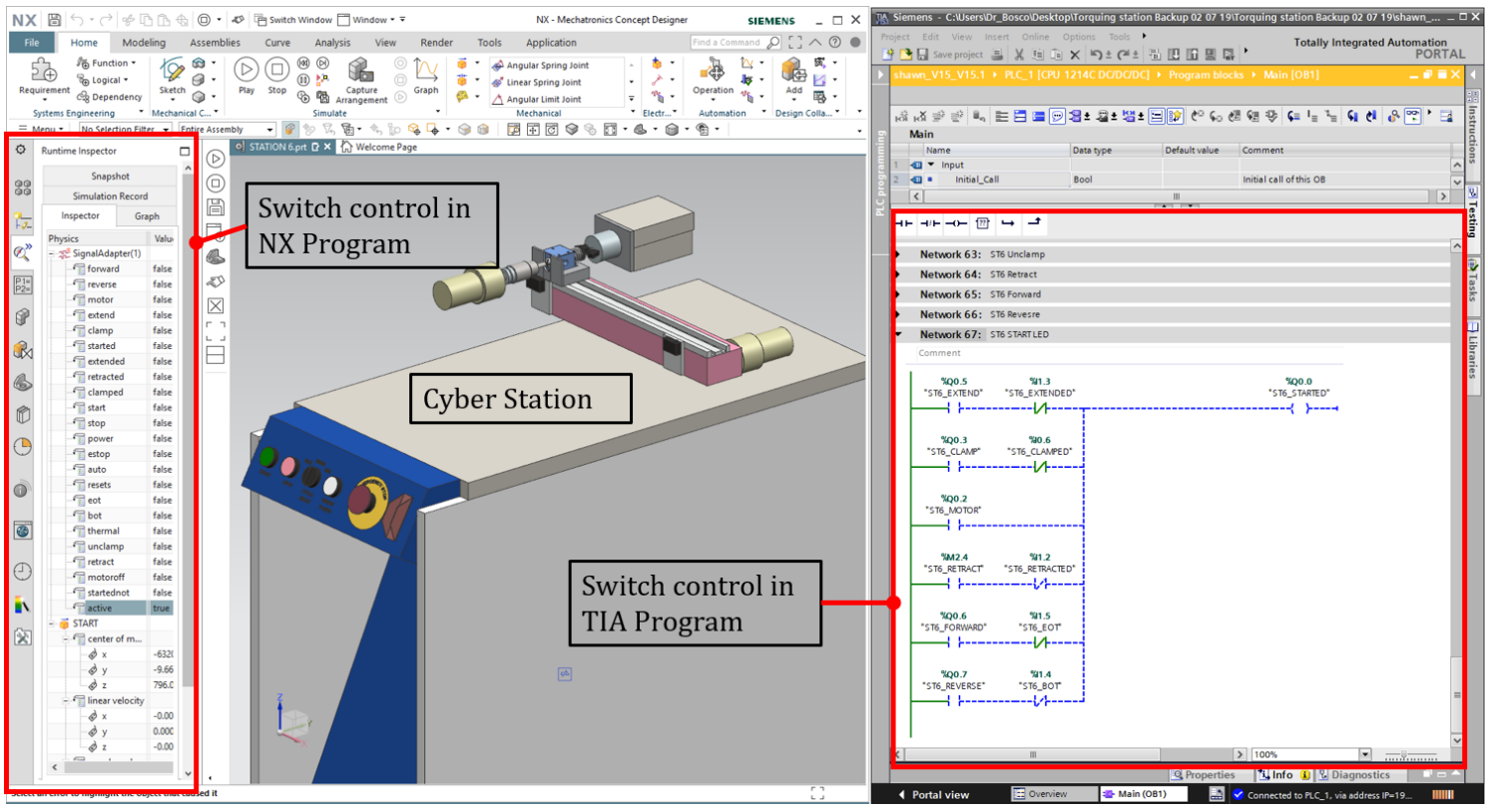

Figure 11. Integrational view of the cyber station in the NX and TIA program.

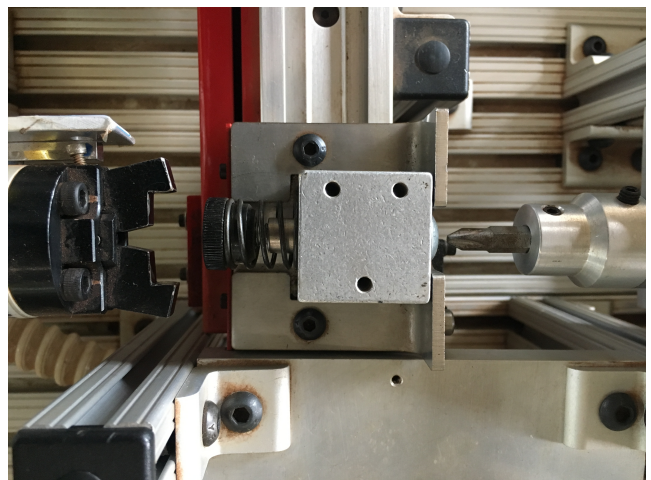

(a)

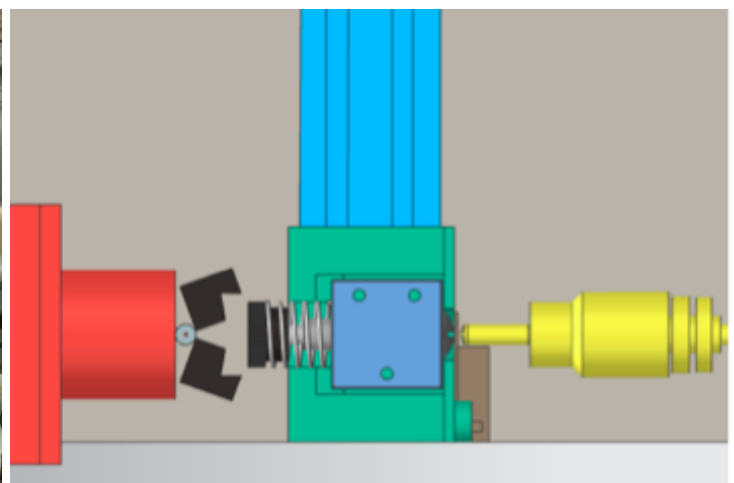

(b)

Figure 12. (a) machine, initial position; (b) virtual model, initial position.

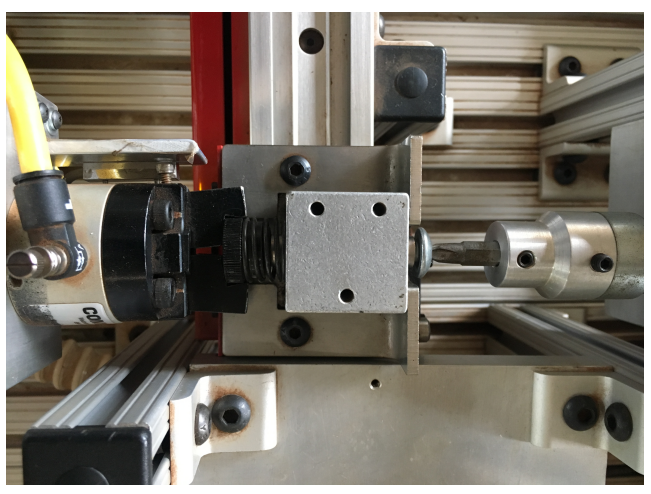

(a)

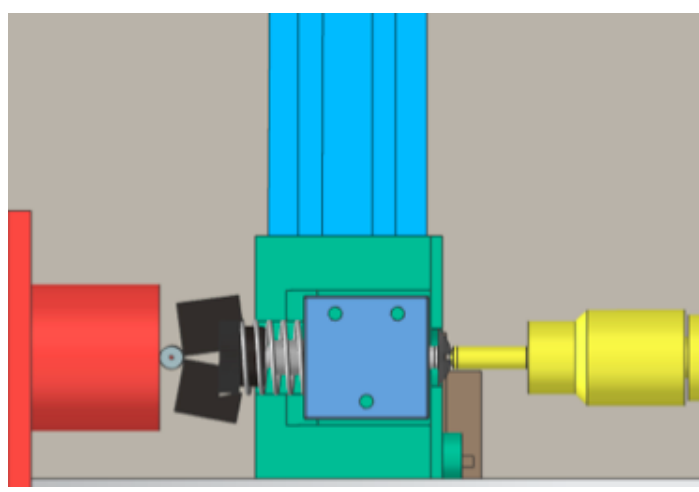

(b)

Figure 13. (a) machine, clamp close; (b) virtual model, clamp close. 


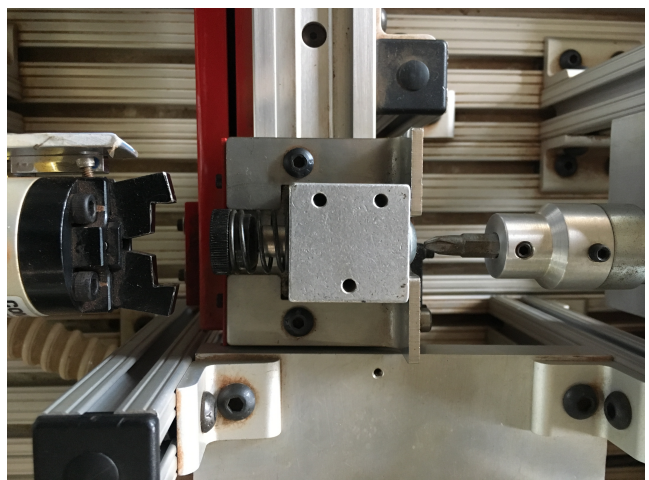

(a)

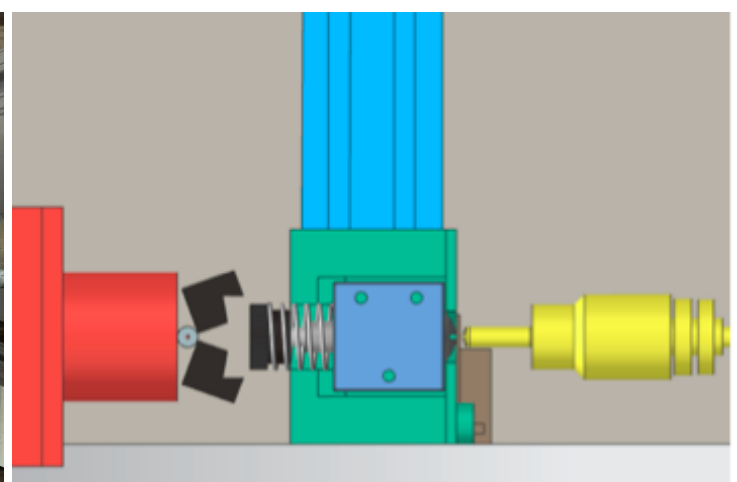

(b)

Figure 14. (a) machine, clamp open after torquing; (b) virtual model, clamp open after torquing.

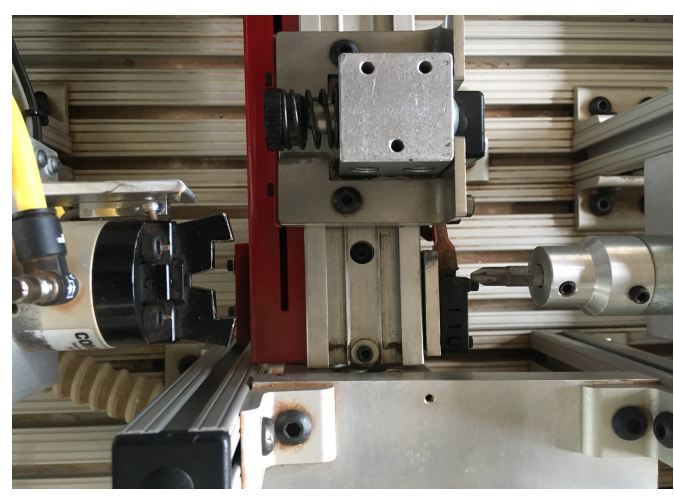

(a)

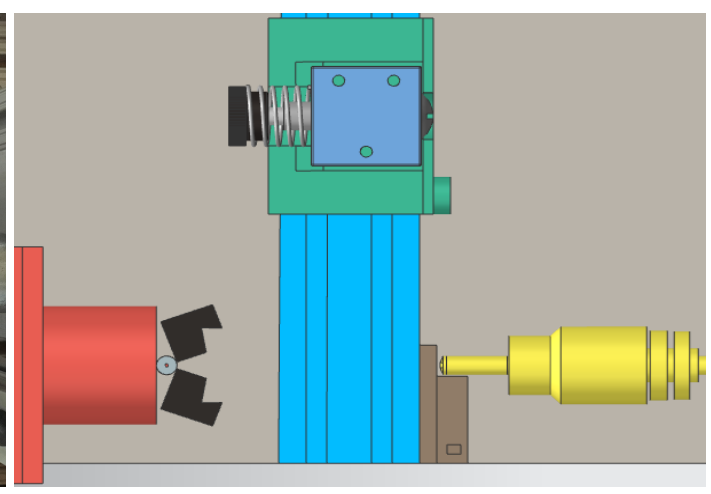

(b)

Figure 15. (a) machine, body in transit to EOT; (b) virtual model, body in transit to EOT.

\subsection{Motion Study}

Upon completion of NX model assembly and communication set-up between the physical system and the digital twin, a motion study was carried out. The aim of the study was to determine the operation time difference between the physical system and virtual model. This study was intended to determine how closely the digital twin reflects the physical system movement. The motion study was done by a series of experiment runs where both systems were run simultaneously. The start and stop times were recorded for each system and the total run time computed and analysed. The experiment set-up consisted of a video recording camera that captures both the physical system and virtual model during each test run. The digital twin was run on a Macintosh laptop: MacBook Air 2012 (Apple Inc, California, U.S.A)with an Intel i5 processor running at a speed of $2.8 \mathrm{GHz}$ with 8 GB RAM memory. The test run video was then analysed to determine the start and stop time and compute the total run time. This experiment was repeated for 10 test runs and the average run time for both systems, the average time lag and time lag standard deviation were computed.

Table 1 shows the time data collected during the experiment and Figure 16 illustrates this data in the form of a graph. From the motion analysis, the average time lag was found to be $0.2880 \mathrm{~s}$ with a standard deviation of $0.3457 \mathrm{~s}$. From observation of the system operation, the time lag was observable but did not adversely affect the system operation. 
Table 1. Motion study experiment data.

\begin{tabular}{cccc}
\hline \multirow{2}{*}{ Runs } & Physical Station & Virtual Station & Time Lag (Error) \\
\cline { 2 - 4 } & Time in Seconds & Time in Seconds & Time in Seconds \\
\hline 1 & 5.39 & 5.95 & 0.56 \\
2 & 5.33 & 4.79 & 0.54 \\
3 & 5.40 & 5.11 & 0.29 \\
4 & 5.35 & 5.02 & 0.33 \\
5 & 5.31 & 5.58 & 0.27 \\
6 & 5.31 & 5.17 & 0.14 \\
7 & 5.33 & 5.20 & 0.13 \\
8 & 5.32 & 5.72 & 0.40 \\
9 & 5.31 & 5.17 & 0.14 \\
10 & 5.21 & 5.21 & 0.08 \\
Mean & 5.2920 & 5.3340 & 0.2880 \\
STD & 0.3501 & 0.0360 & 0.3458 \\
\hline
\end{tabular}

Motion study between the physical and virtual model

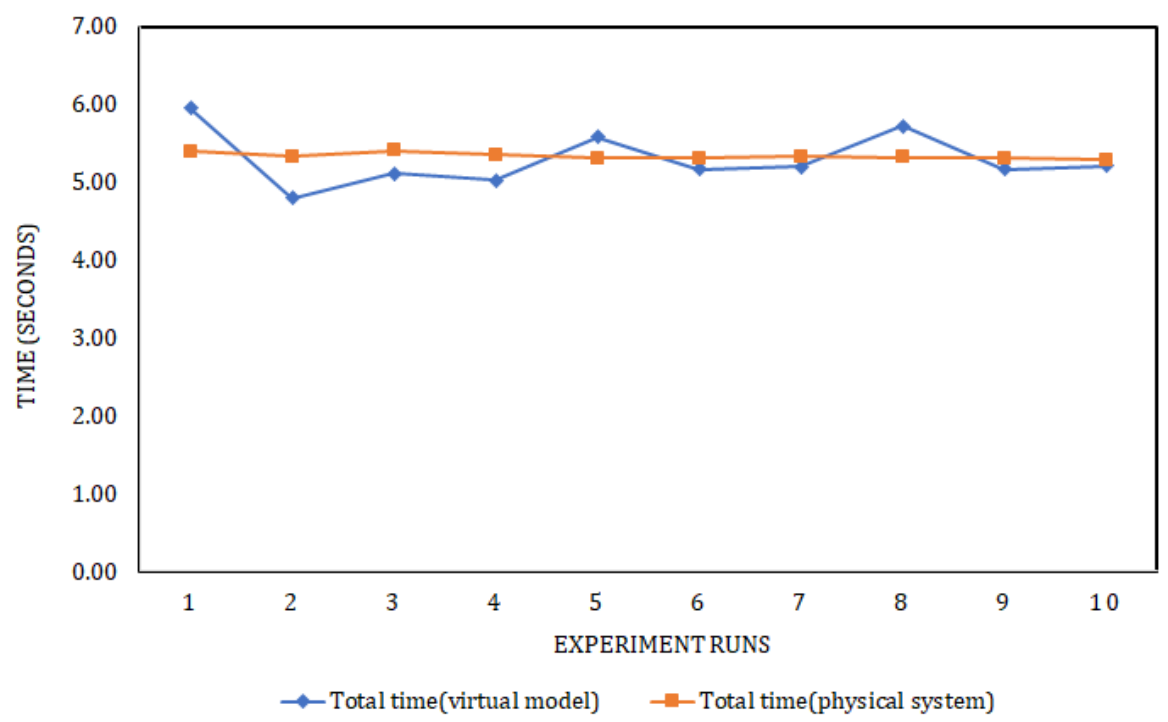

Figure 16. Graph illustrating the run times for the digital twin and physical system for 10 test runs.

A clear source of this time lag was difficult to establish but possible causes considered were the computing hardware used to run the digital twin. Running the digital twin simulation using a higher specification computer might reduce the time lag. It was also considered that the time lag may also be influenced by the communication speed between physical model and digital twin. Currently, the set-up uses CAT5 Ethernet communication cabling with a maximum transfer speed of 1 Gbps with a frequency of $100 \mathrm{MHz}$. A faster communication channel such as CAT6 could improve the time lag. These conjectures will be tested in future motion tests of the system to yield a more conclusive result.

\section{Conclusions}

In light of increasing local and global energy demands, geothermal energy is considered as one of the most promising options. However, high drilling cost limits the quick development and exploration of potential geothermal energy sources. In this paper, a real-time monitoring and control method through the use of a digital twin was proposed for use in geothermal drilling. The concept was implemented on a torquing station of a mechatronic assembly system. The first step in achieving this was the creation of a virtual model, linking the model to its physical counterpart and verification to 
ensure that the functionality of both are synchronized. This goal was fully achieved and tested through the use of Siemens NX and TIA portal which were linked through a KEP-server running the OPC protocol. A future step in this research is the incorporation of sensor data acquisition through cloud computing and analysis of acquired data for optimized control of drilling operations.

Author Contributions: Conceptualization, N.O.O.; Data curation, N.O.O. and M.M.G.; Funding acquisition, J.B.B.; Methodology, N.O.O.; Project administration, J.B.B. and H.S.; Resources, J.B.B.; Supervision, J.B.B.; Validation, H.S.; Writing—original draft, N.O.O. and M.M.G.; Writing—review and editing, N.O.O. and M.G.

Funding: This research received no external funding.

Acknowledgments: This research was carried out at Dedan Kimathi University of Technology (DeKUT)Kenya, Mechatronics laboratory, which offered the equipment required to carry out this research.

Conflicts of Interest: The authors declare no conflict of interest.

\section{References}

1. Lasi, H.; Fettke, P.; Kemper, H.; Feld, T.; Hoffmann, M. Industry 4.0. Bus. Inf. Syst. Eng. 2014, 6, $239-242$. [CrossRef]

2. Qinglin, Q.; Rei, T.; Ying, Z.; Dongming, Z. Digital Twin Service towards Smart Manufacturing. 51st CIRP Conf. Manuf. Syst. 2018, 72, 237-242.

3. Fei, T.; Lasi Meng, Z.; Nee, A.Y.C. Chapter 2: Application of Digital Twin. In Digital Twin Driven Smart Manufacturing; Elsevier: Amsterdam, The Netherlands, 2019; pp. 29-55.

4. Repubic of Kenya, Chapter 4: Assessment of Natural Energy Resources in Kenya. In Updated Least Cost Power Development Plan (Kenya Vision 2030) Study Period: 2017-2037; Government of Kenya: Nairobi, Kenya, 2018; pp. 55-58.

5. Capuano, L. Chapter 5: Geothermal Well Drilling. In Geothermal Power Generation; Capuano Engineering Co: Santa Rosa, CA, USA, 2016; pp. 107-139.

6. Lukawaski, M.; Anderson, B.; Augustine, C.; Capuano, L.; Beckers, K.; Livesay, B.; Tester, J. Cost analysis of oil, gas, and geothermal well drilling. J. Pet. Sci. Eng. 2018, 118, 1-14. [CrossRef]

7. Lyons, W.; Plisga, G.; Lorenz, M. Chapter 4: Drilling and Well completions. In Standard Handbook of Petroleum and Natural Gas Engineering; Elsevier: Amsterdam, The Netherlands, 2015; pp. 1-584.

8. Sammat, E.; O'Shea, S.; Innes, G. Successful Controlled Pressure Drilling Application in a Geothermal Field. In Proceedings of the SPE/IADC Managed Pressured Drilling and Under-balanced Operations Conference and Exhibition, Milan, Italy, 20-21 March 2012.

9. Miyazaki, K.; Ohno, T.; Karasawa, H.; Imaizumi, H. Performance of polycrystalline diamond compact bit based on laboratory tests assuming geothermal well drilling. Geothermics 2019, 80, 185-194. [CrossRef]

10. Cai, Y.; Starley, B.; Cohen, P.; Lee, Y. Sensor data and information fusion to construct digital-twins virtual machine tools for cyber-physical manufacturing. Procedia Manuf. 2017, 10, 1031-1042. [CrossRef]

11. Haag, S.; Anderl, R. Digital twin-Proof of concept. Manuf. Lett. 2018, 15, 64-66. [CrossRef]

(C) 2019 by the authors. Licensee MDPI, Basel, Switzerland. This article is an open access article distributed under the terms and conditions of the Creative Commons Attribution (CC BY) license (http://creativecommons.org/licenses/by/4.0/). 